\title{
Understanding the Role of Internal Diffusion Barriers in Pt/Beta Zeolite Catalyzed Isomerization of $\boldsymbol{n}$-Heptane
}

\author{
Zhongyuan Guo ${ }^{[a]}$, Xin $\mathrm{Li}^{[a]}$, Shen Hu${ }^{[a]}$, Guanghua Ye ${ }^{\star[a]}$, Xinggui Zhou ${ }^{[a]}$, and Marc-Olivier Coppens ${ }^{*[\mathrm{~b}]}$
}

\begin{abstract}
Applications of zeolites in catalysis are plagued by strong diffusion resistance, which results from limitations to molecular transport in micropores, across external crystal surfaces, but also across internal interfaces. The first type of diffusion resistance is well understood, the second is receiving increasing attention, while the diffusion barriers at internal interfaces remain largely unclear. We take Pt/Beta catalyzed isomerization of $n$-heptane as the model system to explore the role of internal diffusion barriers in zeolite catalysis. The two as-synthesized $\mathrm{Pt} /$ Beta catalysts have an identical $P t$ loading, similar Beta particle size and acidity, but different internal structures. A Pt/Beta crystal with no observable internal interfaces can be $180 \%$ higher in activity and $22 \%$ higher in selectivity than its counterpart with numerous internal interfaces. This can only be attributed to the strong transport barriers across internal interfaces, as supported by directly comparing the apparent diffusivities of the two Beta samples.
\end{abstract}

Microporous zeolite catalysts are widely applied in refinery and petrochemical industries, including fluid catalytic cracking, alkylation of aromatics, and isomerization of alkanes. ${ }^{[1,2]}$ The well-defined microporous structure endows zeolites with superior shape selective catalytic properties, on the one hand, but causes strong diffusion resistance, on the other hand. ${ }^{[3]}$ Diffusion limitations are a major problem in improving the activity, selectivity, and stability of zeolitic catalysts. One efficient approach to reduce diffusion limitations is to synthesize zeolites with shortened diffusion path length, such as nano-sized zeolite crystals. $^{[4]}$ However, even when the diffusion path length is shortened to single-unit-cell thickness $(\sim 2 \mathrm{~nm})$, transport limitations could still persist, due to the presence of outersurface and internal diffusion barriers. ${ }^{[5,6]}$ The outer-surface diffusion barriers have been extensively probed by both experiments and simulations, ${ }^{[5-14]}$ while the effects of internal diffusion barriers are much less well documented.

Internal diffusion barriers were discovered when studying mass transfer in very large zeolite crystals $(>10 \mu \mathrm{m}) \cdot{ }^{\left[{ }^{15-19]}\right.}$ In these studies, abnormal mass transfer behavior was observed, e.g., unusual transient concentration profiles. This behavior could only be explained when accounting for the presence of internal diffusion barriers. Such barriers exist on the internal interfaces between intergrowing components of zeolite crystals and originate from mismatches in structure and pore

[a] Z. Guo, X. Li, S. Hu, Dr. G. Ye, Prof. X. Zhou

State Key Laboratory of Chemical Engineering

East China University of Science and Technology

Shanghai 200237 (China)

E-mail: guanghuaye@ecust.edu.cn

[b] Prof. M.-O. Coppens

Department of Chemical Engineering

University College London

London WC1E 7JE (UK)

E-mail: m.coppens@ucl.ac.uk

Supporting information for this article is given via a link at the end of the document. alignment. ${ }^{[20]}$ The aforementioned findings have advanced the understanding of internal diffusion barriers in micron-sized zeolite crystals.

However, for submicron-sized $(0.1-1 \mu \mathrm{m})$ and nano-sized $(<0.1 \mu \mathrm{m})$ zeolites that are of interest to industry, either by themselves or as part of hierarchically structured particles and pellets, knowledge about internal diffusion barriers is very limited. Moreover, the possible influence of these barriers on zeolitecatalyzed reactions has not been reported, except in our recent work. ${ }^{[21]}$ We found that the presence of internal interfaces in submicron-sized zeolites can significantly reduce their apparent activities, which may be attributed to the internal diffusion barriers. Nevertheless, a direct proof of internal diffusion barriers has not been provided, and their role in zeolite-catalyzed reactions has not been revealed.

Here, for the first time, we investigate the role of internal diffusion barriers in Pt/Beta catalyzed isomerization of $n$-heptane. Two Pt/Beta samples were prepared as model catalysts, which possess an identical Pt loading $(0.5 \mathrm{wt} \%)$ and use two Beta zeolites with similar particle size and acidity, but different quantities of internal interfaces. It should be noted that the effect of $\mathrm{Pt}$ sites can be ignored, as the reactions on $\mathrm{Pt}$ sites reach equilibrium very quickly, ${ }^{[22,23]}$ and, thus, the difference in catalytic performance of the model catalysts can be uniquely attributed to the different internal structures of the Beta zeolites. A detailed discussion on the negligible effect of $\mathrm{Pt}$ sites is given in the Supporting Information. The apparent activity and selectivity of the two Pt/Beta catalysts were compared to probe the influence of internal interfaces. Eventually, internal diffusion barriers on the interfaces were demonstrated by analyzing the apparent diffusivities of the Beta samples. The experimental details are given in the Supporting Information.

Fig. 1 illustrates the structure, morphology, and texture of the two as-synthesized Beta samples. The XRD patterns in Fig. 1a display the characteristic peaks of the typical BEA-type structure, indicating the successful synthesis of Beta zeolite. Compared to Beta-B, Beta-A shows a lower peak intensity and its relative crystallinity is calculated to be $91 \%$, suggesting that Beta-A contains a higher fraction of amorphous phase. It is worth noting that any Beta zeolite is considered to be an intergrowth of

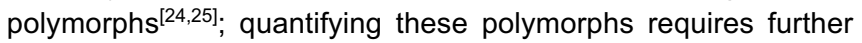
structural characterization that, however, does not impact the comparison between Beta-A and Beta-B. The SEM images in Fig. $1 \mathrm{c}$ and $\mathrm{d}$ show that the two Beta samples possess close average particle sizes ( $240 \mathrm{~nm}$ for Beta-A, $300 \mathrm{~nm}$ for Beta-B), but different morphologies. Beta-A displays very coarse external surfaces and ill-defined external shapes, suggesting that the sample could be polycrystalline, with numerous internal interfaces; Beta-B, in contrast, has smooth external surfaces and well-faceted external shapes, suggesting that the sample could be single-crystalline-like, with few internal interfaces. The HRTEM images and SAED patterns in Fig. $1 \mathrm{e}$ and $f$ prove that Beta-A is polycrystalline, while Beta-B is almost singlecrystalline. The Beta-A particle consists of nano-sized crystals with an average size of $28 \mathrm{~nm}$, and its SAED pattern displays 
diffraction rings, while the Beta-B sample shows ordered lattice fringes and a SAED pattern with bright spots. The $\mathrm{N}_{2}$ adsorption and desorption isotherms in Fig. $1 \mathrm{~b}$ are of Type $\mathrm{I}$, according to the IUPAC classification, for both Beta-A and Beta-B, suggesting negligible mesoporosity in either sample.

Based on the above observations, the Beta samples are both highly crystalline with similar particle size, but different internal structures. For polycrystalline Beta-A, numerous internal interfaces are present, and there are potential mismatches in structure and pore alignment, as well as amorphous phase on these interfaces. ${ }^{[20,26,27]}$ For single-crystalline-like Beta-B, no internal interfaces are observed via the above characterization methods. Some invisible interfaces might exist in both samples $^{[24,25]}$; their possible effect on diffusion is not the focus of this work, but the impact of additional interfaces for Beta-A is.
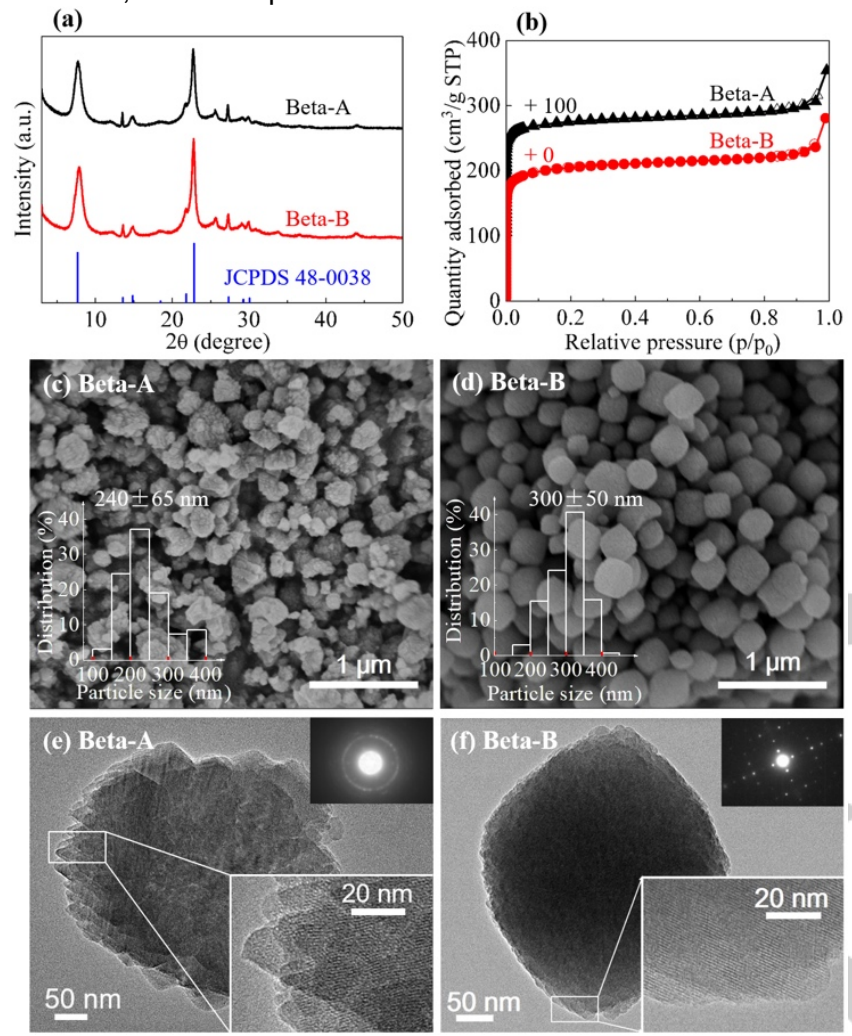

Figure 1. Structure, morphology, and texture of the as-synthesized Beta samples. a) Wide-angle powder XRD patterns; b) $\mathrm{N}_{2}$ adsorption and desorption isotherms; representative SEM images of C) Beta-A and d) Beta-B; representative HRTEM images of e) Beta-A and f) Beta-B, where the inserts with a black background are SAED patterns.

Fig. 2 displays the acidic properties of the two as-synthesized Beta samples. The $\mathrm{NH}_{3}$-TPD curves in Fig. 2a are composed of two desorption peaks, one at low temperature and the other at high temperature. The desorption temperature reflects the acid strength, and the peak area corresponds to the number of acid sites. The numbers of weak and strong acid sites are close for Beta-A and Beta-B, although the number of acid sites in Beta-A is slightly higher. The pyridine-adsorption IR spectra in Fig. $2 \mathrm{~b}$ display bands at 1545,1455 , and $1490 \mathrm{~cm}^{-1}$, which correspond to Brønsted acid sites (BAS), Lewis acid sites (LAS), and the interaction of pyridine with both BAS and LAS, respectively. ${ }^{[28]}$ BAS are believed to be the active sites for $n$-alkanes isomerization; ${ }^{[29]}$ their numbers are summarized in Table 1. The total number of BAS $\left(\mathrm{H}^{+}-473 \mathrm{~K}\right)$ for Beta- $\mathrm{A}$ is $23 \%$ higher than that of Beta-B; the number of strong BAS $\left(\mathrm{H}^{+}-673 \mathrm{~K}\right)$ for Beta-A is $17 \%$ higher. According to the above results, we obtained two Beta samples with similar strengths and amounts of acid sites.
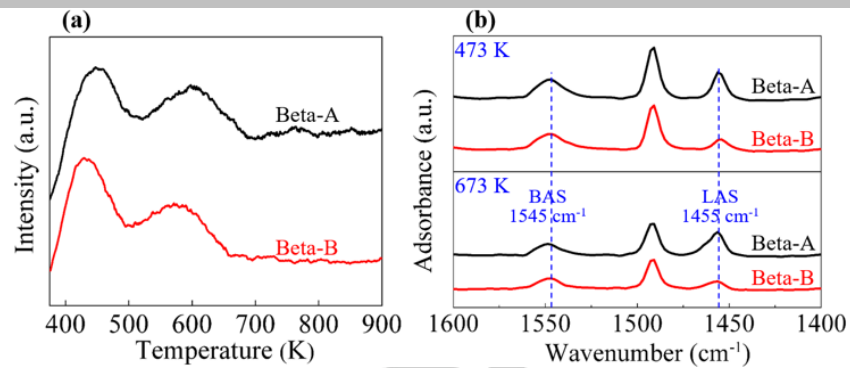

Figure 2. Acidity of the as-synthesized Beta samples. a) $\mathrm{NH}_{3}-\mathrm{TPD}$ profiles of the Beta samples; b) Py-IR curves of Beta-A and Beta-B.

Table 1. Some characteristics of the two Beta zeolites synthesized in this work. More characteristics are listed in Tables S2 and S3 in the Supporting Information.

\begin{tabular}{cccccc}
\hline Samples & ${\text { Si } / A \mathrm{~A}^{\left[{ }^{[a]}\right]}}^{\text {Crystal }}$ & $\begin{array}{c}\text { Particle } \\
\text { size } \mathrm{e}^{[\mathrm{b}]}(\mathrm{nm})\end{array}$ & $\begin{array}{c}\mathrm{H}^{+}-473 \mathrm{~K}^{[\mathrm{d}]} \\
(\mathrm{mmol} / \mathrm{g})\end{array}$ & $\begin{array}{c}\mathrm{H}^{+}-673 \mathrm{~K}^{[\mathrm{d}]}(\mathrm{nm}) \\
(\mathrm{mmol} / \mathrm{g})\end{array}$ \\
\hline Beta-A & 24 & 28 & 240 & 0.161 & 0.084 \\
Beta-B & 25 & 300 & 300 & 0.131 & 0.072 \\
\hline
\end{tabular}

[a] Si/AI molar ratio measured by using ICP-AES. [b] Crystal size measured from HRTEM images. [c] Particle size measured from SEM images. [d] Brønsted acid content measured from Py-IR spectra; $\mathrm{H}^{+}-473 \mathrm{~K}$ and $\mathrm{H}^{+}-673 \mathrm{~K}$ are measured at 473 and $673 \mathrm{~K}$, respectively.

The two Pt/Beta catalysts were tested in a fixed-bed reactor under the following conditions: $T=515-568 \mathrm{~K}, P=1 \mathrm{~atm}, W H S V$ $=40.8 \mathrm{~g}_{n \text {-heptane }}\left(\mathrm{g}_{\text {cat }} \mathrm{h}\right)^{-1}, \mathrm{H}_{2} / n$-heptane mole $=23.5$. The catalytic performance of the two catalysts is displayed in Fig. 3. As seen from Figs. 3a-e, at $515 \mathrm{~K}$, the conversions of $n$-heptane for $\mathrm{Pt} /$ Beta-A are slightly higher than the ones for Pt/Beta-B, and their selectivities to isomer products are almost identical. For example, at $515 \mathrm{~K}$, the conversion at $t=1 \mathrm{~h}$ for Pt/Beta-A is $13 \%$ higher than that for $\mathrm{Pt} / \mathrm{Beta} \mathrm{B}$. When the temperature increases to $529 \mathrm{~K}$, both conversion and selectivity for Pt/Beta-B become higher than those for Pt/Beta-A. At $568 \mathrm{~K}$, the conversion and selectivity at $t=1 \mathrm{~h}$ for Pt/Beta-B are $128 \%$ and $22 \%$ higher than these for Pt/Beta-A, respectively. At low temperatures $(T \leq 515$ $\mathrm{K}$ ), the apparent reaction rate is controlled by reaction, and, thus, this rate is determined by the acidity of the catalyst. The number of BAS for Beta-A is $23 \%$ higher than that for Beta-B, which explains the slightly higher conversions for Pt/Beta-A at $515 \mathrm{~K}$. At high temperatures $(T \geq 529 \mathrm{~K})$, the intrinsic reaction rates are so high that the diffusion path length and diffusion resistance significantly affect the apparent reaction rates. Since the two catalysts use Beta zeolites with similar particle size, the much lower conversions for Pt/Beta-A at $568 \mathrm{~K}$ should be attributed to the stronger diffusion resistance. There are numerous observable internal interfaces in Beta-A, which may generate additional diffusion resistance. The lower selectivity for Pt/BetaA can also be attributed to stronger diffusion limitations that lead to undesired cracking side reactions of isomer products.

To compare the activities of the two catalysts on the same basis, the turnover frequencies (TOFs) based on the total number of BAS $\left(\mathrm{H}^{+}-473 \mathrm{~K}\right)$ are determined and summarized in the Arrhenius plot (see Fig. 3f). The TOFs of Pt/Beta-B can be $180 \%$ higher than those of $P t / B e t a-A$ at the temperature of 568 $\mathrm{K}$. Besides, the apparent activation energy for Pt/Beta-A (62 $\mathrm{kJ} / \mathrm{mol})$ is much lower than that for Pt/Beta-B (103 kJ/mol). The diffusion resistance in Pt/Beta-A is much higher, which reduces the apparent activation energy. ${ }^{[30]}$ 


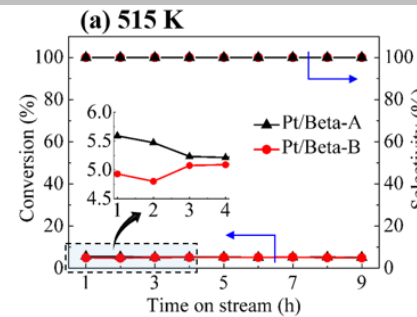

(c) $538 \mathrm{~K}$

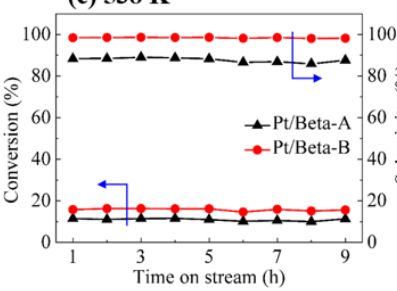

(e)
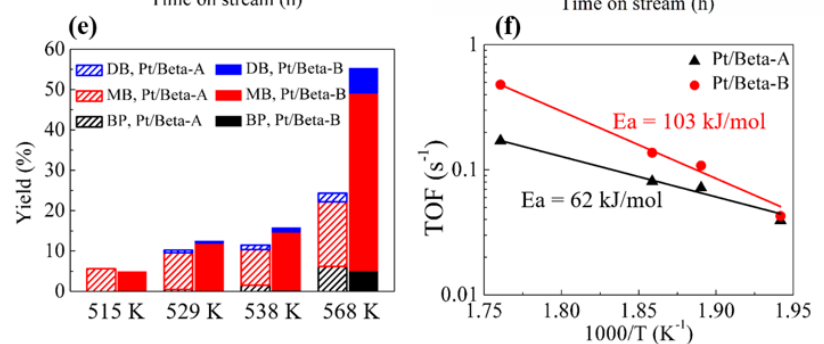

(b) $529 \mathrm{~K}$

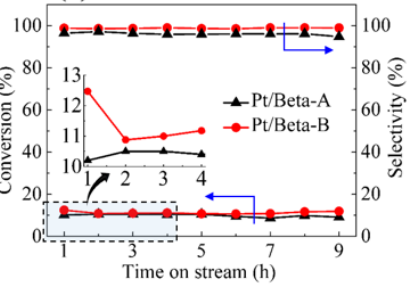

(d) $568 \mathrm{~K}$

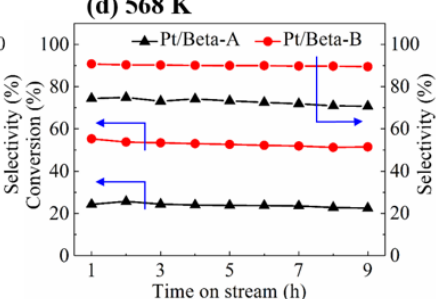

Figure 3. Catalytic performance of Pt/Beta-A and Pt/Beta-B. Conversion of $n$ heptane and selectivity to isomers at the temperatures of a) $515 \mathrm{~K}$, b) $529 \mathrm{~K}, \mathrm{c}$ ) $538 \mathrm{~K}$, and d) $568 \mathrm{~K}$; e) yields of di-branched isomers (DB), mono-branched isomers, and by-products (BP) at a time on stream of $1 \mathrm{~h}$. Yields at different times on stream are given in Fig. S5 in the Supporting Information; f) Arrhenius plot of the TOFs at a time on stream of $1 \mathrm{~h}$. The other reaction conditions are as follows: $\mathrm{P}=1 \mathrm{~atm}, \mathrm{WHSV}=40.8 \mathrm{~g}_{n \text {-heptane }}\left(\mathrm{g}_{\text {cat }} \mathrm{h}\right)^{-1}, \mathrm{H}_{2} / n$ heptane molar ratio $=23.5$.

To present direct proof of the significant internal diffusion barriers in Beta-A, we compared the apparent diffusivities of $n$ heptane in Beta-A and Beta-B, determined by the zero length column method (ZLC), as seen in Fig. $4 .^{[31]}$ In the temperature range of 393-443 K, the apparent diffusivities in Beta-A are $48 \%$ $71 \%$ lower than the ones in Beta-B, and the apparent activation energy for Beta-A (54 kJ/mol) is $46 \%$ higher than that for Beta-B (37 kJ/mol). Apparently, there exist strong activation energy barriers on the internal interfaces of Beta-A, which generate additional diffusion resistance. A similar result relating to energy barriers is also found in very large zeolite $X$ crystals $(>10 \mu \mathrm{m})$ containing defects. ${ }^{[15]}$ It should be noted that the height of the internal diffusion barriers during catalytic tests cannot be inferred from that during ZLC measurements. During catalytic tests, multiple components are present, and the temperature and the loading of $n$-heptane are higher. Evaluating these effects on internal diffusion barriers is the subject of future work.
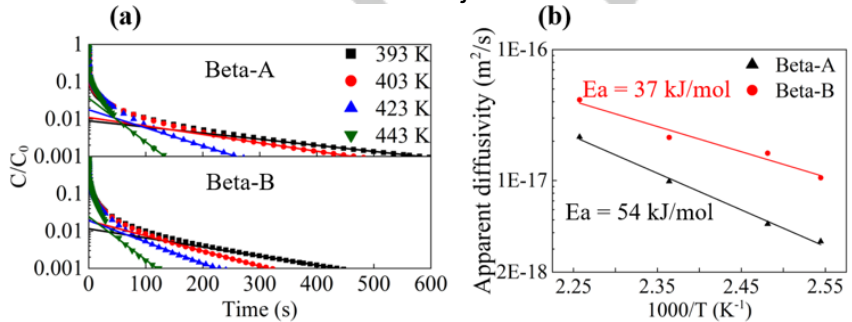

Fig. 4. Apparent diffusivities of $n$-heptane $\left(D_{a p p}\right)$ measured by the zero length column (ZLC) method. ${ }^{[31]}$ a) Desorption curves; b) Arrhenius plot of apparent diffusivities. Flow rate $100 \mathrm{~mL} / \mathrm{min}$, atmospheric pressure. When calculating the apparent diffusivities, the two samples are assumed to be spheres with

radii $(R)$ equal to half of their particle sizes (see Table 1 ). The inverses of the diffusion time constants $\left(D_{a p p} / R^{2}\right)$ are given in Table S5 in the Supporting Information.

In conclusion, we compared the catalytic performance of two model Pt/Beta catalysts to explore how the internal diffusion barriers affect the Pt/Beta catalyzed isomerization of $n$-heptane. $\mathrm{Pt} /$ Beta-A and $\mathrm{Pt} /$ Beta-B use Beta zeolites with similar particle size and acidity but distinct internal structures. Beta-A contains numerous internal interfaces, while no observable internal interfaces were found in Beta-B. The internal interfaces can generate additional diffusion barriers that limit the transport of reactant and products in Beta zeolites. Due to the smaller number of internal diffusion barriers, the Pt/Beta- $B$ sample is $180 \%$ more active, and $22 \%$ more selective towards isomer products. This work has discussed a strategy to explore the internal diffusion barriers in submicron/nano-sized zeolites, and the results clearly show that the role of internal diffusion barriers should be accounted for when developing zeolitic materials for catalysis.

\section{Acknowledgements}

This work was supported by the National Natural Science Foundation of China (21706067), the "Chenguang Program" supported by Shanghai Education Development Foundation and Shanghai Municipal Education Commission (17CG29), and the Fundamental Research Funds for the Central Universities (222201714004 and 222201718003). M.-O.C is supported by the EPSRC "Frontier Engineering" Centre for Nature Inspired Engineering (EP/K038656/1) and the UK Catalysis Hub (EP/K014706/1).

\section{Conflict of interest}

The authors declare no conflict of interest.

Keywords: Zeolite catalysis • internal diffusion barriers $•$ Pt/Beta -Isomerization of $n$-heptane $\cdot$ interfaces

[1] M. Hartmann, A. G. Machoke, W. Schwieger, Chem. Soc. Rev. 2016, 45, 3313-3330.

[2] A. Corma, Chem. Rev. 1997, 97, 2373-2419.

[3] B. Smit, T. L. M. Maesen, Nature 2008, 451, 671-678.

[4] Y. Wei, T. E. Parmentier, K. P. De Jong, J. Zečević, Chem. Soc. Rev. 2015, 44, 7234-7261.

[5] P. Bai, E. Haldoupis, P. J. Dauenhauer, M. Tsapatsis, J. I. Siepmann, ACS Nano 2016, 10, 7612-7618.

[6] C. C. Chang, A. R. Teixeira, C. Li, P. J. Dauenhauer, W. Fan, Langmuir 2013, 29, 13943-13950.

[7] O. C. Gobin, S. J. Reitmeier, A. Jentys, J. A. Lercher, J. Phys. Chem. C 2009, 113, 20435-20444.

[8] S. J. Reitmeier, O. C. Gobin, A. Jentys, J. A. Lercher, Angew. Chemie - Int. Ed. 2009, 48, 533-538.

[9] J. Kärger, T. Binder, C. Chmelik, F. Hibbe, H. Krautscheid, R. Krishna, J. Weitkamp, Nat. Mater. 2014, 13, 333-343. 
[10] N. E. R. Zimmermann, B. Smit, F. J. Keil, J. Phys. Chem. C 2010, 114, 300-310.

[11] J. C. Saint Remi, A. Lauerer, C. Chmelik, I. Vandendael, H. Terryn G. V. Baron, J. F. M. Denayer, J. Kärger, Nat. Mater. 2016, 15, $401-$ 406.

[12] N. E. R. Zimmermann, S. P. Balaji, F. J. Keil, J. Phys. Chem. C 2012, 116, 3677-3683.

[13] F. Hibbe, C. Chmelik, L. Heinke, S. Pramanik, J. Li, D. M. Ruthven, D. Tzoulaki, J. Kärger, J. Am. Chem. Soc. 2011, 133, 2804-2807.

[14] G. Ye, Z. Guo, Y. Sun, K. Zhu, H. Liu, X. Zhou, M.-O. Coppens, Chemie Ing. Tech. 2017, 89, 1333-1342.

[15] A. Feldhoff, J. Caro, H. Jobic, J. Ollivier, C. B. Krause, P. Galvosas, J. Kärger, ChemPhysChem 2009, 10, 2429-2433.

[16] S. Vasenkov, W. Böhlmann, P. Galvosas, O. Geier, H. Liu, J. Kärger, J. Phys. Chem. B 2001, 105, 5922-5927.

[17] S. Vasenkov, J. Kärger, Microporous Mesoporous Mater. 2002, 55, 139-145.

[18] O. Geier, S. Vasenkov, E. Lehmann, J. Kärger, U. Schemmert, R. A. Rakoczy, J. Weitkamp, J. Phys. Chem. B 2001, 105, 10217-10222.

[19] E. R. Geus, J. C. Jansen, H. van Bekkum, Zeolites 1994, 14, 82-88.

[20] L. Karwacki, M. H. F. Kox, D. a M. de Winter, M. R. Drury, J. D. Meeldijk, E. Stavitski, W. Schmidt, M. Mertens, P. Cubillas, N. John, et al., Nat. Mater. 2009, 8, 959-965.

[21] G. Ye, Y. Sun, Z. Guo, K. Zhu, H. Liu, X. Zhou, M. O. Coppens, J. Catal. 2018, 360, 152-159.

[22] D. Jin, G. Ye, J. Zheng, W. Yang, K. Zhu, M.-O. Coppens, X. Zhou, ACS Catal. 2017, 7, 5887-5902.

[23] T. Yue, W. Liu, L. Li, X. Zhao, K. Zhu, X. Zhou, W. Yang, J. Catal. 2018, 364, 308-327.

M. M. J. Treacy, J. M. Newsam, Nature 1988, 332, 249-251.

[25] A. Cantín, A. Corma, M. J. Díaz-Cabanas, J. L. Jorda, M. Moliner, F. Rey, Angew. Chemie Int. Ed. 2006, 45, 8013-8015.

[26] E. Stavitski, M. R. Drury, D. A. M. De Winter, M. H. F. Kox, B. M. Weckhuysen, Angew. Chemie - Int. Ed. 2008, 47, 5637-5640. Z. Liu, T. Ohsuna, K. Sato, T. Mizuno, T. Kyotani, T. Nakane, O. Terasaki, Chem. Mater. 2006, 18, 922-927. A. Astafan, M. A. Benghalem, Y. Pouilloux, J. Patarin, N. Bats, C. Bouchy, T. J. Daou, L. Pinard, J. Catal. 2016, 336, 1-10. H. D. Setiabudi, A. A. Jalil, S. Triwahyono, J. Catal. 2012, 294, 128 135. M. Höchtl, A. Jentys, H. Vinek, J. Catal. 2000, 190, 419-432. 
Entry for the Table of Contents (Please choose one layout)

Layout 1:

\section{COMMUNICATION}

Diffusion barriers on interfaces: There exist very strong internal diffusion barriers in Beta zeolites, which subsequently reduce their activity and selectivity in catalysing isomerization of $n$-heptane. The Beta zeolite with no-observable internal interfaces can be $180 \%$ higher in activity and $22 \%$ higher in selectivity.

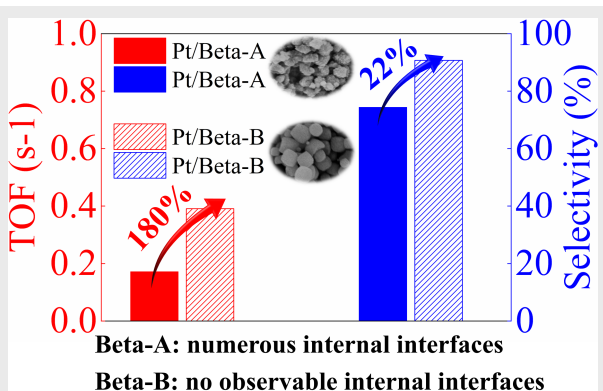

Zhongyuan Guo, Xin Li, Shen Hu, Guanghua Ye*, Xinggui Zhou, and MarcOlivier Coppens*

Page No. - Page No.

Understanding the Role of Internal Diffusion Barriers in Pt/Beta Catalyzed Isomerization of $\boldsymbol{n}$-Heptane 